\title{
Transplante de membrana amniótica em casos agudos graves de queimadura ocular química e síndrome de Stevens-Johnson
}

\author{
Amniotic membrane transplantation for severe acute cases of chemical ocular \\ burn and Stevens-Johnson syndrome
}

\author{
José Reinaldo da Silva Ricardo ${ }^{1}$ \\ Sabrina Leite de Barros ${ }^{2}$ \\ Myrna Serapião dos Santos ${ }^{3}$ \\ Luciene Barbosa deSouza ${ }^{4}$ \\ José Álvaro Pereira Gomes ${ }^{5}$
}

Trabalho realizado no Departamento de Oftalmologia da Universidade Federal de São Paulo - UNIFESP - São Paulo (SP) - Brasil.

${ }^{1}$ Médico estagiário do Setor de Córnea e Doenças Oculares Externas do Departamento de Oftalmologia da Universidade Federal de São Paulo - UNIFESP - São Paulo (SP) - Brasil.

${ }^{2}$ Médica estagiária do Setor de Córnea e Doenças Oculares Externas do Departamento de Oftalmologia da UNIFESP - São Paulo (SP) - Brasil.

${ }^{3}$ Médica colaboradora do Setor de Córnea e Doenças Oculares Externas do Departamento de Oftalmologia da UNIFESP - São Paulo (SP) - Brasil.

${ }^{4}$ Doutora em Oftalmologia e Chefe do Setor de Córnea e Doenças Externas do Departamento de Oftalmologia da UNIFESP - São Paulo (SP) - Brasil.

${ }^{5}$ Doutor em Oftalmologia e Professor afiliado do Setor de Córnea e Doenças Externas do Departamento de Oftalmologia da UNIFESP - São Paulo (SP) - São Paulo - Brasil.

Endereço para Correspondência: José Reinaldo da Silva Ricardo. Rua Apiacás, 600 - Apto. 123 - São Paulo (SP) CEP 05017-020

E-mail: reinaldoricardo@bol.com.br

Recebido para publicação em 24.07.2008

Última versão recebida em 06.01.2009

Aprovação em 19.02.2009

Nota Editorial: Depois de concluída a análise do artigo sob sigilo editorial e com a anuência do Dr. José Augusto Alves Ottaiano sobre a divulgação de seu nome como revisor, agradecemos sua participação neste processo.

\begin{tabular}{l} 
RESUMO \\
\hline Objetivo: Investigar o potencial terapêutico do transplante de membrana \\
amniótica nos casos agudos graves de queimadura ocular química e \\
síndrome de Stevens-Johnson. Métodos: Foram analisados retrospecti- \\
vamente os prontuários de oito pacientes, com um total de dez olhos, \\
submetidos a transplante de membrana amniótica para tratamento de \\
queimadura ocular química e síndrome de Stevens-Johnson na fase aguda \\
entre janeiro de 1999 e maio de 2008 no Departamento de Oftalmologia \\
daUNIFESP.Dados referentes a sexo,idade, grau da queimadura, etiologia, \\
olho acometido, achados oftalmológicos, extensão da membrana \\
amniótica, operações adicionais, tempo em dias entre a lesão e a cirurgia, \\
acuidade visual antes e depois da cirurgia, defeito epitelial em dias, \\
complicações e tempo de seguimento em meses foram coletados. Resul- \\
tados: A idade média dos pacientes foi de $35,7 \pm 23,04$ anos, dos quais \\
seis eram homens e dois eram mulheres. Três pacientes (quatro olhos) \\
apresentaram síndrome de Stevens-Johnson e cinco pacientes (seis olhos) \\
apresentaram queimadura ocular química. O defeito epitelial foi cicatri- \\
zado em média de $27,8 \pm 4,7$ dias (variando de 20 a 35 dias). Todos os \\
pacientes evoluíram com deficiência límbica em seguimento médio de \\
$7,8 \pm 2,8$ meses (variando entre cinco e doze meses) e quatro olhos \\
desenvolveram simbléfaro. Conclusões: Os resultados sugerem que o \\
transplante de membrana amniótica representa um aditivo que pode ser \\
realizado nos casos agudos graves de queimadura ocular química e \\
síndrome de Stevens-Johnson coma finalidade de promovera epitelização \\
e suprimir a inflamação e suas consequências, se comparado a outros \\
trabalhos que trataram casos semelhantes com terapia medicamentosa \\
somente. Por outro lado, nãoé capaz de evitar a deficiência límbica nesses \\
casos, que futuramente necessitarão de transplante de células-tronco do \\
limbo ou outras cirurgias para correção da superfície ocular. \\
\hline
\end{tabular}

Descritores: Âmnio/transplante; Síndrome de Stevens-Johnson; Córnea; Epitélio anterior; Queimaduras oculares

\section{INTRODUÇÃO}

O tratamento da fase aguda das queimaduras oculares químicas e da síndrome de Stevens-Johnson (SSJ) consiste em promover a epitelização, reduzir a inflamação e prevenir o "melting” tecidual progressivo. Embora a fisiopatogenia das doenças sejam completamente diferentes, o objetivo 
do tratamento é o mesmo: evitar o ciclo vicioso de complicações que podem levar a sequelas como cicatrizes conjuntivais/corneais e perda visual grave ${ }^{(1-3)}$. O tratamento é clínico nos casos mais leves de queimadura ocular química (graus I e II de Roper-Hall ${ }^{(4)}$ ), porém nos mais graves (graus III e IV de Roper-Hall) as medidas clínicas não são suficientes para prevenir as complicações indesejadas e os pacientes acabam perdendo a visão ${ }^{(1)}$. Nestes casos de prognóstico reservado, têm-se ultimamente utilizado a membrana amniótica (MA) para o recobrimento da superfície queimada no intuito de diminuir a inflamação, facilitar a epitelização, evitar o "melting" de córnea e esclera, bem como diminuir a neovascularização corneana e a cicatrização exacerbada da conjuntiva ${ }^{(5)}$.

Em um estudo multicêntrico ${ }^{(5)}$ foi descrito os resultados do transplante de MA em 13 olhos de 11 pacientes com queimadura aguda (10 queimaduras químicas e 3 térmicas), dos quais 7 eram casos de queimadura graus II-III e 6 casos grau IV, pela classificação de Roper-Hall. Os autores observaram epitelização completa em $85 \%$ dos casos e melhora da acuidade visual dos pacientes com queimadura grau II e III de Hoper-Hall. Todos os olhos com queimadura grau IV desenvolveram deficiência total de células-tronco límbicas.

Na SSJ, o tratamento clínico pouco ajuda a diminuir a inflamação ocular intensa da fase aguda e a reconstrução da superfície na fase crônica é difícil. Recentemente, o transplante de MA na superfície ocular durante a fase aguda tem mostrado grande expectativa por diminuir a inflamação e suas sequelas $^{(6-7)}$. Outros autores ${ }^{(8)}$ analisaram o uso da MA em pacientes com deficiência de células-tronco límbicas, entre eles casos de SSJ, e chegaram à conclusão de que para deficiência parcial do limbo, o transplante de MA isolado era suficiente para restaurar o epitélio corneano, mas para deficiência total do limbo, era necessário associar transplante autólogo do limbo.

As ações da MA têm sido estudadas em diferentes modelos experimentais in vitro e in vivo e incluem: estímulo à epitelização e inibição da inflamação, neovascularização e fibrose. Um estudo comparou a intensidade da inflamação num modelo experimental de queimadura por álcali tratado ou não com transplante de $\mathrm{MA}^{(9)}$. Foi observado menor intensidade de infiltração por polimorfonucleares, epitelização mais rápida e menor opacidade no grupo tratado com MA, principalmente quando usada com face epitelial para baixo. Outra importante evidência que se soma às outras ações da MA compreende a presença dos inibidores das metaloproteinases teciduais (TIMS), que foram encontrados nas células epiteliais e mesenquimais do âmnion ${ }^{(10-12)}$.

Baseando-se nestes estudos e na nossa experiência clínica com MA, foi investigado o potencial terapêutico do transplante de membrana amniótica, realizado na fase aguda, nos casos graves de queimadura ocular química e síndrome de Stevens-Johnson.

\section{MÉTODOS}

\section{Pacientes}

Foram analisados retrospectivamente os prontuários de oito pacientes, com um total de dez olhos, submetidos a transplante de MA para tratamento de queimadura ocular química e SSJ na fase aguda entre janeiro de 1999 e maio de 2008 no Departamento de Oftalmologia da UNIFESP.

Dados referentes a sexo, idade, grau da queimadura, etiologia, olho acometido, achados oftalmológicos, extensão da MA, operações adicionais, tempo entre a lesão e a cirurgia em dias, acuidade visual antes e depois da cirurgia, defeito epitelial em dias, complicações e tempo de seguimento em meses foram coletados.

\section{Captação e preservação da MA}

A obtenção e o processamento da MA foram realizados de acordo com as normas do protocolo aprovado pela Comissão de Ética da UNIFESP/EPM.

A MA foi obtida a partir das placentas provenientes de cesáreas eletivas realizadas no Hospital Maternidade Amparo Maternal após consentimento assinado pelas gestantes. Todas essas pacientes tiveram exames sorológicos negativos para HIV-1, hepatite B (HBsAg) e sífilis (VDRL), que foram reconfirmados pela realização de sorologia do sangue do cordão umbilical após o parto.

Após a obtenção da placenta no centro cirúrgico obstétrico, procedeu-se à sua lavagem com o uso de solução fisiológica 0,9\% em ambiente estéril. Posteriormente, o âmnio foi separado do córion com a utilização de tesoura e pinça estéreis e estendido sobre um filtro de nitrocelulose estéril (Millipore, Bedfort, MA, EUA) com a face epitelial para cima. A membrana e o filtro foram lavados com solução tampão fosfato contendo $1.000 \mathrm{U} / \mathrm{ml}$ de penicilina, $20 \mathrm{mcg} / \mathrm{ml}$ de estreptomicina e $2,5 \mathrm{mcg} / \mathrm{ml}$ de anfotericina B (Ophthalmos, São Paulo), cortados em fragmentos de aproximadamente $3 \times 3 \mathrm{~cm}$, colocados em um recipiente estéril com o meio TC-199: glicerol 1:1 (Ophthalmos, São Paulo) e congelados a $-80^{\circ} \mathrm{C}$.

\section{Técnica cirúrgica}

A técnica cirúrgica utilizada foi descrita originalmente por Brown ${ }^{(13)}$ (Figura 1A e B). Como ilustrado, a MA foi suturada nas margens palpebrais e nos fórnices como um "patch" para cobrir a área lesada. Um ou dois cortes de MA $(3 \times 4 \mathrm{~cm})$ foi retirado do papel filtro de nitrocelulose e transferido para o olho receptor para cobrir toda a superfície ocular da margem da pálpebra superior até a margem da pálpebra inferior. A MA foi suturada na conjuntiva e na margem palpebral utilizando sutura contínua com mononylon 9.0 ou 10.0 (Ethicon Inc.) ou polivicryl 8-0 (Ethicon Inc.). Nos fórnices superiores e inferiores a MA foi suturada com fio de seda 6.0 (Ethicon Inc.) temporal e nasal, e presa na pele com protetores de pele de borracha ou de silicone. 

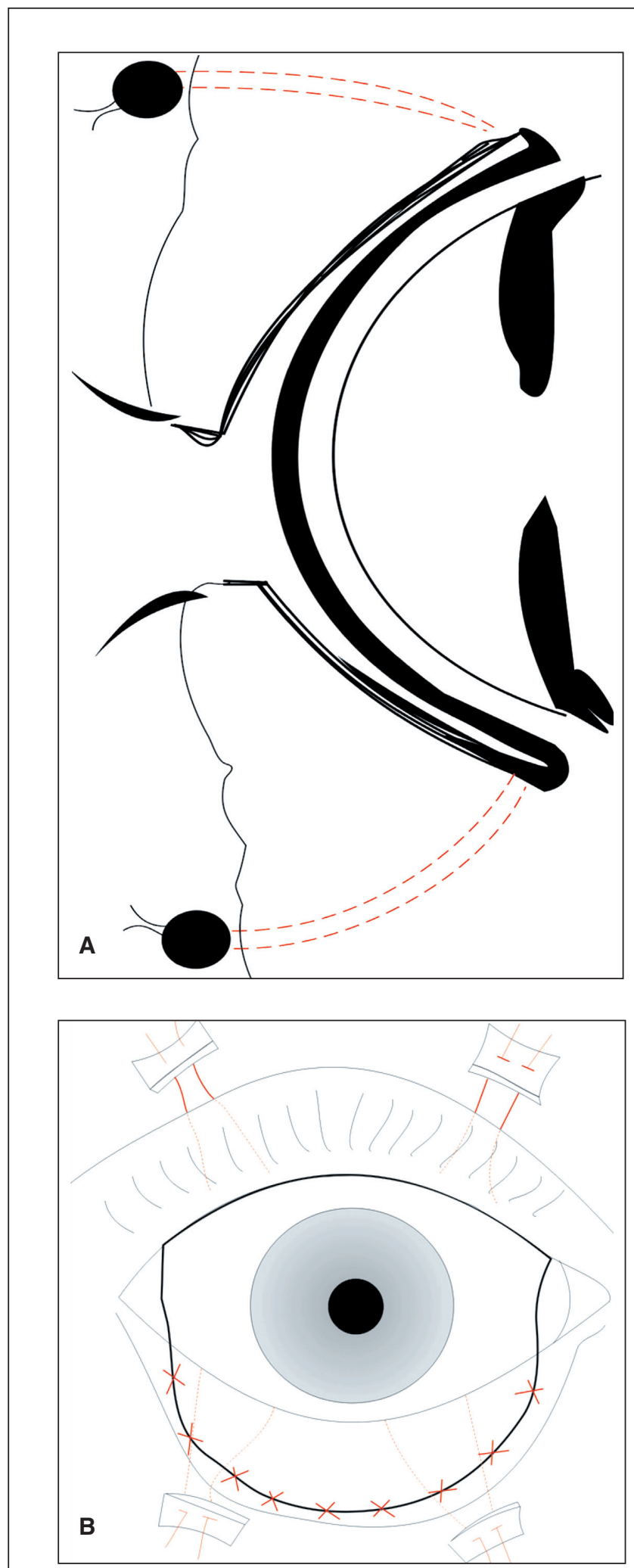

Figura 1 - Técnica de Brown: A) Figura esquemática mostrando como o transplante de membrana amniótica cobre a superfície ocular inteira da margem palpebral superior à margem palpebral inferior; B) A membrana amniótica fica presa na pele com protetores de silicone e na margem palpebral com sutura contínua ou pontos separados
Nos casos que foram realizados tarsorrafias laterais, a lamela anterior e posterior da margem palpebral foi separada na linha cinzenta com um bisturi $\mathrm{n}^{\circ}$ 11. A borda conjuntival da margem palpebral posterior foi removida com tesoura. Passava-se uma sutura contínua com categute 6-0 unindo as lamelas posteriores das duas pálpebras, uma segunda sutura em "U" unindo as lamelas anteriores das pálpebras superiores e inferiores com nylon ancorado em pedaços de esponja ou silicone.

\section{RESULTADOS}

A idade dos pacientes variou entre 9 e 76 anos $(35 \pm 23,04$ anos), dos quais 6 eram homens e 2 eram mulheres. Três pacientes apresentaram SSJ (Figura 2), dos quais 4 olhos foram submetidos a transplante de MA e 5 pacientes apresentaram queimadura ocular química por álcalis (4 por cal e 2 por soda cáustica), dos quais 6 olhos foram submetidos ao mesmo procedimento.

Todos os olhos com queimadura ocular química apresentaram classificação grau IV de Roper-Hall ${ }^{(4)}$. Os achados clínicos encontram-se resumidos na tabela 1.

Foi realizado o transplante de MA, recobrindo córnea, fórnix e conjuntiva tarsal até a linha cinzenta, conforme descrito originalmente por Brown ${ }^{(13)}$. Nos olhos 1,2 e 5 também foi realizada tarsorrafia lateral. Nos olhos 1,8 e 10 foi realizado transplante tectônico associado durante o mesmo ato cirúrgico, pois o defeito epitelial crônico evoluiu com perfuração corneana. O olho 7 necessitou de recobrimento conjuntival na esclera por afinamento escleral.

O transplante de MA foi realizado entre 10 e 30 dias (20,5 \pm 7,6 dias) depois da lesão. A acuidade visual antes e após a cirurgia encontra-se resumida na tabela 1.

Notavelmente, a dor foi aliviada imediatamente após o transplante de MA em todos os pacientes. O defeito epitelial depois

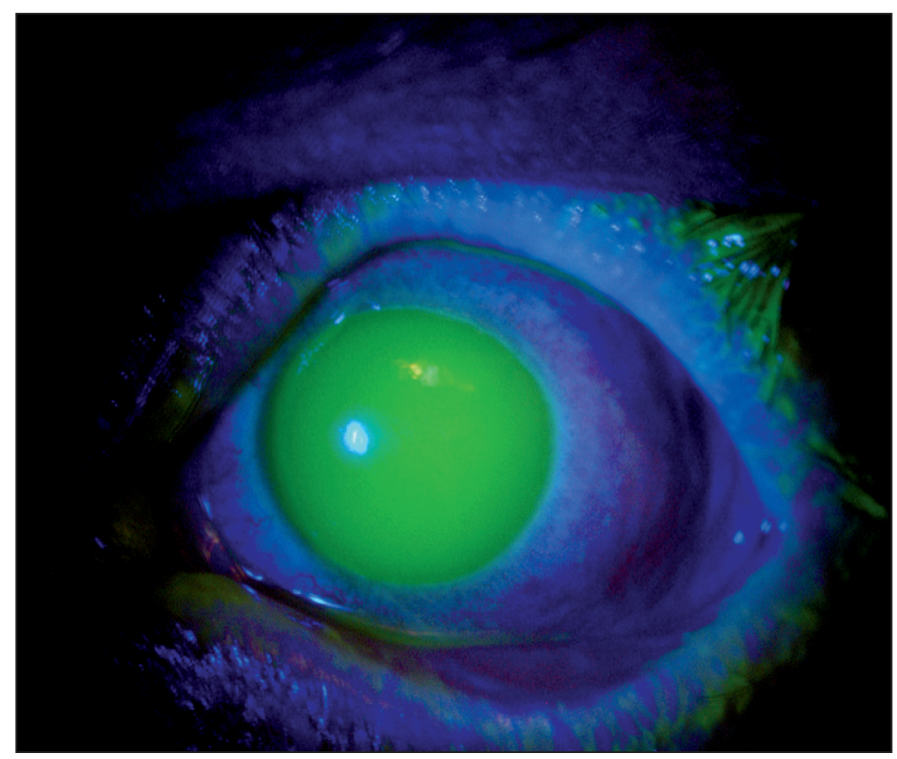

Figura 2 - Coloração com fluoresceína confirmando o grande defeito epitelial da córnea em um paciente com SSJ na fase aguda no caso 4 


\begin{tabular}{|c|c|c|c|c|c|c|c|c|c|c|c|}
\hline Olho & $\begin{array}{l}\text { Sexo/ } \\
\text { idade } \\
\text { (anos) }\end{array}$ & $\begin{array}{l}\text { Agente/ } \\
\text { olho }\end{array}$ & Grau & Achados & $\begin{array}{c}\text { Extensão } \\
\text { da MA }\end{array}$ & $\begin{array}{l}\text { Operações } \\
\text { adicionais }\end{array}$ & $\begin{array}{l}\text { Tempo entre } \\
\text { lesão e } \\
\text { cirurgia } \\
\text { (dias) }\end{array}$ & $\begin{array}{c}\text { AV } \\
\text { antes/ } \\
\text { depois }\end{array}$ & $\begin{array}{c}\text { Defeito } \\
\text { epitelial } \\
\text { (dias) }\end{array}$ & $\begin{array}{c}\text { Necrose/ } \\
\text { simbléfaro/ } \\
\text { DL }\end{array}$ & $\begin{array}{c}\text { Seguimento } \\
\text { (meses) }\end{array}$ \\
\hline 1 & $\begin{array}{l}\mathrm{M} / \\
57\end{array}$ & $\begin{array}{l}\text { Cal/ } \\
\text { OD }\end{array}$ & IV & $\begin{array}{l}\text { DC } 100 \% \text {, } \\
\text { IL } 70 \%, \\
\text { HC } 4+, \text { PO }\end{array}$ & $\begin{array}{c}\text { Total + } \\
\text { TT + } \\
\text { TL }\end{array}$ & $\begin{array}{l}\text { Simbp + } \\
M L+G S\end{array}$ & 30 & $\begin{array}{c}\mathrm{PL} / \\
\mathrm{CD} 1,5 \mathrm{~m}\end{array}$ & 25 & $\begin{array}{c}\text { Simbléfaro/ } \\
\text { DL }\end{array}$ & 6 \\
\hline 2 & $\begin{array}{l}\mathrm{M} / \\
57\end{array}$ & $\begin{array}{l}\mathrm{Cal} / \\
\mathrm{OE}\end{array}$ & IV & $\begin{array}{l}\text { DC } 100 \% \text {, } \\
\text { IL } 50 \% \text {, } \\
\text { HC } 4+\end{array}$ & $\begin{array}{c}\text { Total }+ \\
\text { TL }\end{array}$ & $\begin{array}{l}\text { Simbp + } \\
M L+G S\end{array}$ & 30 & $\begin{array}{l}\mathrm{PL} / \\
\mathrm{PL}\end{array}$ & 25 & $\begin{array}{c}\text { Simbléfaro/ } \\
\text { DL }\end{array}$ & 6 \\
\hline 3 & $\begin{array}{l}\mathrm{M} / \\
20\end{array}$ & $\begin{array}{l}\text { SSJ/ } \\
\text { OD }\end{array}$ & - & $\begin{array}{l}\text { DC } 90 \% \text {, } \\
\text { IL } 60 \% \text {, } \\
\text { HC } 4+\end{array}$ & Total & MA & 15 & $\begin{array}{l}\mathrm{MM} / \\
20 / 40\end{array}$ & 30 & $\mathrm{DL}$ & 5 \\
\hline 4 & $\begin{array}{l}M / \\
20\end{array}$ & $\begin{array}{l}\text { SSJ/ } \\
\text { OE }\end{array}$ & - & $\begin{array}{l}\text { DC } 100 \% \text {, } \\
\text { IL } 70 \% \text {, } \\
\text { HC } 4+\end{array}$ & Total & MA & 15 & $\begin{array}{l}\mathrm{MM} / \\
20 / 32\end{array}$ & 35 & $\mathrm{DL}$ & 5 \\
\hline 5 & $\begin{array}{c}\mathrm{M} / \\
9\end{array}$ & $\begin{array}{l}\text { SSJ/ } \\
\text { OD }\end{array}$ & - & $\begin{array}{l}\text { DC } 90 \% \text {, } \\
\text { IL } 50 \% \text {, } \\
\text { HC } 4+\end{array}$ & $\begin{array}{c}\text { Total }+ \\
\text { TL }\end{array}$ & $\mathrm{TL}$ & 15 & $\begin{array}{c}\mathrm{MM} / \\
\mathrm{CD} 2 \mathrm{~m}\end{array}$ & 20 & $\mathrm{DL}$ & 12 \\
\hline 6 & $\begin{array}{l}\mathrm{M} / \\
42\end{array}$ & $\begin{array}{l}\mathrm{Cal} / \\
\mathrm{OD}\end{array}$ & IV & $\begin{array}{l}\text { DC } 100 \% \text {, } \\
\text { IL } 90 \% \text {, } \\
\text { HC } 4+, A C\end{array}$ & Córnea & $\begin{array}{l}\text { TT + } \\
\text { MA }\end{array}$ & 15 & $\begin{array}{l}1,0 / \\
\text { MM }\end{array}$ & 28 & $\mathrm{DL}$ & 12 \\
\hline 7 & $\begin{array}{l}F / \\
76\end{array}$ & $\begin{array}{l}\text { Soda } \\
\text { cáustica/ } \\
\text { OD }\end{array}$ & IV & $\begin{array}{l}\text { DC } 100 \% \text {, } \\
\text { IL } 90 \% \text {, } \\
\text { HC } 4+, \text { AE }\end{array}$ & $\begin{array}{c}\text { Córnea + } \\
\text { RC na } \\
\text { esclera }\end{array}$ & - & 25 & $\begin{array}{c}20 / 80 / \\
\text { MM }\end{array}$ & 25 & Simbléfaro & 5 \\
\hline 8 & $\begin{array}{l}\mathrm{M} / \\
11\end{array}$ & $\begin{array}{l}\text { Soda } \\
\text { cáustica/ } \\
\text { OD }\end{array}$ & IV & $\begin{array}{l}\text { DC } 80 \% \text {, } \\
\text { IL } 50 \% \text {, } \\
\text { HC } 4+\end{array}$ & $\begin{array}{l}\mathrm{TT}+ \\
\mathrm{MA}\end{array}$ & - & 20 & $\begin{array}{l}\mathrm{MM} / \\
\mathrm{MM}\end{array}$ & 25 & $\begin{array}{c}\text { Simbléfaro/ } \\
\text { DL }\end{array}$ & 8 \\
\hline 9 & $\begin{array}{l}\mathrm{M} / \\
35\end{array}$ & $\begin{array}{l}\mathrm{Cal} / \\
\mathrm{OD}\end{array}$ & IV & $\begin{array}{l}\text { DC } 90 \% \text {, } \\
\text { IL } 70 \% \text {, } \\
\text { HC } 4+\end{array}$ & MA & - & 10 & $\begin{array}{l}\text { CD } 1,5 \mathrm{~m} / \\
\text { CD1 m }\end{array}$ & 35 & $\mathrm{DL}$ & 9 \\
\hline 10 & $\begin{array}{l}\mathrm{F} / \\
30\end{array}$ & $\begin{array}{l}\text { SSJ/ } \\
\text { OD }\end{array}$ & - & $\begin{array}{l}\text { DC } 80 \% \text {, } \\
\text { IL } 70 \% \text {, PO }\end{array}$ & $\begin{array}{c}\mathrm{MA}+ \\
\mathrm{TT}\end{array}$ & $\begin{array}{c}\text { "Patch" } \\
\text { córnea + MA }\end{array}$ & 30 & $\begin{array}{l}0,1 / \\
\text { MM }\end{array}$ & 30 & $\mathrm{DL}$ & 10 \\
\hline
\end{tabular}

da cirurgia foi cicatrizado em todos os pacientes em média de $27,8 \pm 4,7$ dias (variou de 20 e 35 dias). Todos os pacientes evoluíram com deficiência límbica total (Figura 3) em um seguimento médio de 7,8 $\pm 2,8$ meses (variando de 5 a 12 meses) e quatro olhos (40\%) desenvolveram simbléfaro (1, 2, 7 e 8).

Nos olhos 1 e 2 foi posteriormente realizada simblefaroplastia, implante de mucosa labial e implante de glândula salivar para reconstrução de fundo de saco e tratamento de olho seco. Nos olhos 3 e 4 foi repetido o transplante de MA por novo episódio de SSJ. O olho 5 foi submetido a transplante de limbo para correção de deficiência límbica. Os olhos 6 e 10 foram posteriormente submetidos a transplante de córnea e MA e "patch" de córnea e MA, respectivamente, por perfuração ocular secundária à deficiência total de células-tronco límbicas após seis meses de pós-operatório.

\section{DISCUSSÃO}

Neste estudo, foi avaliado o potencial terapêutico do transplante de MA para o tratamento de casos agudos graves de SSJ e queimaduras oculares químicas. Importante salientar a dificuldade em realizar um estudo prospectivo comparativo neste grupo de pacientes pelo pequeno número de casos e diversidade de variáveis envolvidas. A mesma observação foi relatada por outros autores ${ }^{(5)}$.

Esses resultados mostram que o transplante de MA pode ser considerado um procedimento cirúrgico para promover a epitelização e prevenir sequelas cicatriciais se comparado com a terapia medicamentosa realizada em casos semelhantes aos deste trabalho, conforme relatado por outros autores ${ }^{(14-15)}$. De acordo com esses autores, o transplante de MA realizado na fase aguda das queimaduras oculares químicas grau II e III da classificação de Roper-Hall resultou em epitelização e alívio da dor mais rápido que o tratamento medicamentoso realizado em queimaduras com a mesma graduação, e nos casos mais graves, como os dos pacientes deste estudo, o tempo de epitelização, o alívio da dor e as complicações foram semelhantes, não apresentando diferenças estatisticamente significantes.

Em outro trabalho, foi descrito uma série de quatro casos de queimadura ocular grau IV de Roper-Hall que foram 


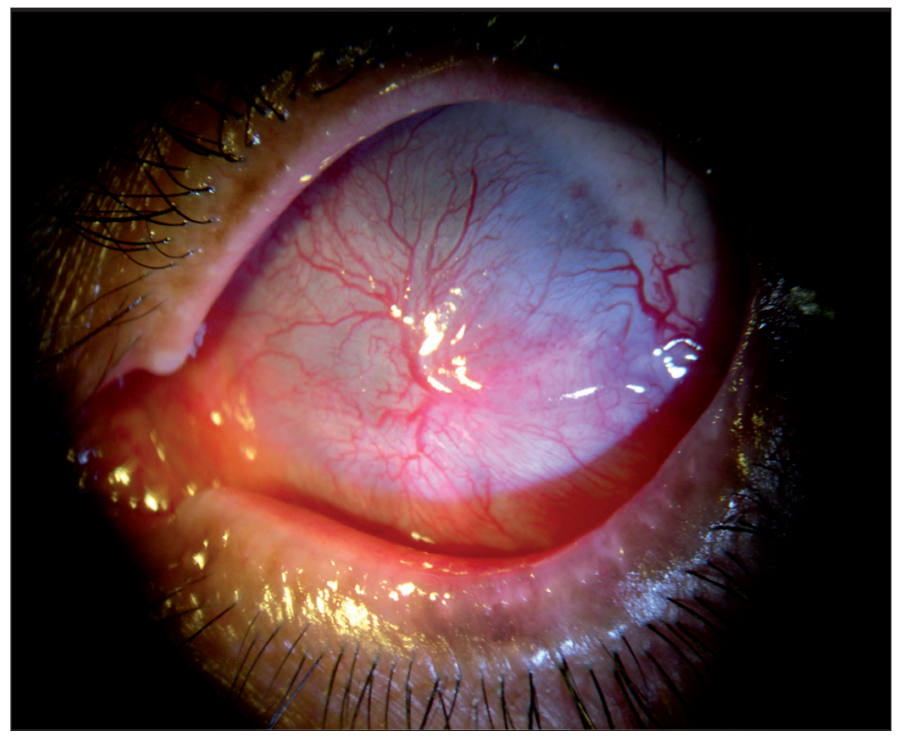

Figura 3 - Deficiência límbica total seis meses após queimadura ocular química no caso 1

tratados com transplante de MA na fase aguda ${ }^{(16)}$. Nesse estudo foi observado falência em todos os casos, dos quais dois olhos sofreram evisceração espontânea, um foi submetido à evisceração posterior por dor crônica e outro entrou em phthisis bulbi. Não houve nenhuma dessas complicações nos nossos pacientes, o que está de acordo com estudos anteriores ${ }^{(5)}$. É importante ressaltar que os casos descritos por esses autores ${ }^{(16)}$ apresentavam isquemia mais grave, com $360^{\circ}$ de isquemia límbica. Isto reflete a extrema gravidade das queimaduras oculares nesses pacientes e chama a atenção para a inacurácia da classificação de Roper-Hall $^{(4)}$ em distinguir graus diferentes de gravidade nos casos mais graves. Em 2001, foi proposta uma nova classificação ${ }^{(17)}$ para queimadura química em seis diferentes níveis de gravidade que leva em consideração não somente a área de isquemia, mas também o grau de acometimento conjuntival pela coloração com fluoresceína. Talvez se os casos tivessem sido classificados de acordo com a classificação essa nova classificação as diferenças encontradas entre os resultados deste trabalho e os de Joseph et al. pudessem ser mais bem entendidas.

Sequelas (como entrópio, simbléfaro e olho seco) geram um ciclo vicioso que levam a dano ocular no estágio crônico e representa mais dificuldade para reconstrução da superfície ocular. A MA reduz essas sequelas tanto por via indireta pela sua ação antiinflamatória e pela promoção do crescimento epitelial corneano quanto por via direta pelo mecanismo anticicatrizante do lado estromal da membrana, que atua inibindo a sinalização do fator de crescimento beta, a proliferação e a diferenciação de miofibroblastos ${ }^{(18)}$. Estes efeitos explicam a relativa baixa incidência de formação de simbléfaro notada quando MA foi transplantada na fase $\operatorname{aguda}^{(19-20)}$.

\section{CONCLUSÃO}

Os resultados sugerem que o transplante de MA representa um aditivo que pode ser realizado nos casos agudos graves de queimadura ocular química e SSJ com a finalidade de promover a epitelização e suprimir a inflamação e suas consequências, se comparado a outros trabalhos que trataram casos semelhantes com terapia medicamentosa somente.

Por outro lado, não é capaz de evitar a deficiência límbica nesses casos, que futuramente necessitarão de transplante células-tronco do limbo ou outras cirurgias para correção da superfície ocular.

\section{ABSTRACT}

Purpose: To study the therapeutic potential of amniotic membrane transplantation in cases of severe acute chemical ocular burn and Stevens-Johnson syndrome. Methods: We retrospectively reviewed the records of eight patients, with a total of ten eyes, submitted to amniotic membrane transplantation for treatment of ocular chemical burns and Stevens-Johnson syndrome in the acute phase between January 1999 and May 2008 in the Ophthalmology Department of UNIFESP. Data relating to sex, age, degree of chemical burns, etiology, affected eye, ophthalmological findings, extension of amniotic membrane, surgeries, additional time between the injury and surgery in days, visual acuity before and after surgery, epithelial defect healing (days), complications and time of follow-up in months were collected. Results: The average age of patients was $35.7 \pm$ 23.04 years, with six men and two women. Three patients (four eyes) had Stevens-Johnson syndrome and five patients (six eyes) had chemical ocular burn. The epithelial defect was healed at an average of $27.8 \pm 4.7$ days (ranging from 20 and 35 days). All patients presented limbal stem cell deficiency in a median follow-up of $7.8 \pm 2.8$ months (ranging from six and twelve months) and four eyes developed symblepharon. Conclusions: The results suggest that the amniotic membrane transplantation represents an additive that can be carried out in the serious cases of ocular chemical burn and StevensJohnson syndrome with the finality of promoting the epithelialization and abolishing the inflammation and its consequences, if compared with other studies that treated similar cases with medical therapy only. On the other hand, it is not possible to avoid the limbic deficiency in these cases, which in the future will need limbal stem cell transplantation or other surgeries for correction of the ocular surface.

Keywords: Amnion/transplantation; Stevens-Johnson syndrome; Cornea; Epithelium, corneal; Eye burns

\section{REFERÊNCIAS}

1. Wagoner MD. Chemical injuries of the eye: current concepts in pathophysiology and therapy. Surv Ophthalmol. 1997;4(4)1:275-313. 
2. Pfister RR. Chemical corneal burns. Int Ophthalmol Clin. 1984;24(2):157-68.

3. Pires JL, Pires RT, Gomes JA, Glória MA, Tseng SC. Transplante de membrana amniótica para reconstrução da superfície ocular. In: Gomes JA, Alves MR, editores. Superfície ocular: córnea, limbo, conjuntiva, filme lacrimal. Rio de Janeiro: Cultura Médica; 2006. p.293-306.

4. Roper-Hall MJ. Thermal and chemical burns. Trans Ophthalmol Soc UK. 1965;85:631-53.

5. Meller D, Pires RT, Mack RJ, Figueiredo F, Heiligenhaus A, Park WC, et al. Thermal and chemical burns. Ophthalmology. 2000;107:980-89.

6. Gregory DG. The ophthalmologic management of acute Stevens-Johnson syndrome. Ocul Surf. 2008;6(2):87-95.

7. Di Pascuale MA, Espana EM, Liu DT, Kawakita T, Li W, Gal YY, et al. Correlation of corneal complications with eyelid cicatricial pathologies in patients with Stevens-Johnson syndrome and toxic epidermal necrolysis syndrome. Ophthalmology. 2005;112(5):904-12.

8. Tseng SC, Prabhasawat P, Barton K, Gray T, Meller D. Amniotic membrane transplantation with or without limbal allografts for corneal surface reconstruction in patients with limbal stem cell deficiency. Arch Ophthalmol. 1998; 116(4):431-41

9. Kim JS, Kim JC, Na BK, Jeong JM, Song CY. Amniotic membrane patching promotes healing and inhibits proteinase activity on wound healing following acute corneal alkali burn. Exp Eye Res.2000;70(3):329-37.

10. Gomes JA, Romano A, Santos MS, Dua HS. Amniotic Membrane use in Ophthalmology. Curr Opin Ophthalmol. 2005;16:233:40.

11. Dua HS, Gomes JA, King AJ, Maharajan VS. The amniotic membrane in ophthalmology. Surv Ophthalmol. 2004;49(1):51-77.

12. Rowe TF, King LA, MacDonald PC, Casey ML. Tissue inhibitor of metal- loproteinase-1 and tissue inhibitor of metalloproteinase-2 expression in human amnion mesenchymal and epithelial cells. Am J Obstet Gynecol. 1997;176(4): 915-21.

13. Brown AL. Lime Burns of the eye: use of the rabbit peritoneum to prevent severe delayed effects. Experimental studies and report of cases. Arch Ophthalmol. 1941;26:754-69.

14. Tamhane A, Vajpayee R, Biswas NR, Pandey RM, Sharma N, Titiyal JS, et al. Evaluation of amniotic membrane transplantation as an adjunct to medical therapy as compared with medical therapy alone in acute ocular burns. Ophthalmoloy. 2005;112(11):1963-9.

15. Prabhasawat P, Tesavibul N, Prakairungthong N, Booranapong W. Efficacy of amniotic membrane patching for acute chemical and thermal burns. J Med Assoc Thai. 2007;90(2):319-26.

16. Joseph A, Dua HS, King AJ. Failure of amniotic membrane transplantation in the treatment of acute ocular burns. Br J Ophthalmol. 2001;85(9):1065-9. Comment in: Br J Ophthalmol. 2001;85(7):831.

17. Dua HS, King AJ, Joseph A. A new classification of ocular surface burns. Br J Ophthalmol. 2001;85(11):1379-83. Comment in: Br J Ophthalmol. 2004;88(10): 1353-4; author reply $1354-5$.

18. Tseng SC, Li DQ, Ma X. Suppression of transforming growth factor-beta isoforms, TGF-beta receptor type II, and myofibroblast differentiation in cultured human corneal and limbal fibroblasts by amniotic membrane matrix. J Cell Physiol. 1999;179(3):325-35.

19. Sorsby A, Symons HM. Amniotic membrane grafts in caustic burns of the eye. (Burn of the second degree). Br J Ophthalmol. 1946;30(6):337-45.

20. Sorsby A, Haythorne J, Reed H. Further experience with amniotic membrane graft in caustic burns of the eye. Br J Ophthalmol. 1947;31(7):409-18. 